\title{
SELECTION PRINCIPLES AND SPECIAL SETS OF REALS
}

\author{
BOAZ TSABAN
}

\begin{abstract}
We give a selection of major open problems involving selective properties, diagonalizations, and covering properties for sets of real numbers.

This is a revision of the version published as a chapter in the book Open Problems in Topology II (E. Pearl, ed.), Elsevier B.V., 2007, 91-108. The present version reports solutions of some problems, uses up-to-date notation, and has updated bibliography.

Comments and further updates would be appreciated.
\end{abstract}

\section{INTRODUCTION}

The field of Selective Principles in Mathematics started with Scheepers' identification and classification of common prototypes for selective properties appearing in classical and modern works. For surveys of the field see [58, 31, 69].

The main four prototypes in the field are defined as follows. Fix a topological space $X$, and let $\mathscr{A}$ and $\mathscr{B}$ each be a collection of covers of $X$. Following are properties which $X$ may or may not have [51].

$\left(\begin{array}{l}\mathscr{A} \\ \mathscr{B}\end{array}\right)$ : Every member of $\mathscr{A}$ has a subset which is a member of $\mathscr{B}$.

$\mathrm{S}_{1}(\mathscr{A}, \mathscr{B})$ : For each sequence $\left\{\mathcal{U}_{n}\right\}_{n \in \mathbb{N}}$ of members of $\mathscr{A}$, there exist members $U_{n} \in \mathcal{U}_{n}, n \in \mathbb{N}$, such that $\left\{U_{n}: n \in \mathbb{N}\right\} \in \mathscr{B}$.

$\mathrm{S}_{\text {fin }}(\mathscr{A}, \mathscr{B})$ : For each sequence $\left\{\mathcal{U}_{n}\right\}_{n \in \mathbb{N}}$ of members of $\mathscr{A}$, there exist finite subsets $\mathcal{F}_{n} \subseteq \mathcal{U}_{n}, n \in \mathbb{N}$, such that $\bigcup_{n \in \mathbb{N}} \mathcal{F}_{n} \in \mathscr{B}$.

$\mathrm{U}_{\text {fin }}(\mathscr{A}, \mathscr{B})$ : For each sequence $\left\{\mathcal{U}_{n}\right\}_{n \in \mathbb{N}}$ of members of $\mathscr{A}$ which do not contain a finite subcover, there exist finite subsets $\mathcal{F}_{n} \subseteq \mathcal{U}_{n}, n \in \mathbb{N}$, such that $\left\{\cup \mathcal{F}_{n}: n \in \mathbb{N}\right\} \in \mathscr{B}$.

When $\mathscr{A}$ and $\mathscr{B}$ vary through topologically significant collections, we obtain properties studied in various contexts by many authors. We give some examples.

Fix a topological space $X$, and let $\mathrm{O}$ denote the collection of all open covers of $X$. In the case of metric spaces, $\mathrm{S}_{\text {fin }}(\mathrm{O}, \mathrm{O})$ is the property shown by Hurewicz [27] to be equivalent to Menger's basis property

Supported by the Koshland Center for Basic Research. 
[40], and $\mathrm{S}_{1}(\mathrm{O}, \mathrm{O})$ is Rothberger's property traditionally known as $C^{\prime \prime}$ [48], a property related to Borel's strong measure zero [14].

Considering special types of covers we obtain additional properties. Henceforth, by cover of $X$ we mean a nontrivial one, i.e., such that $X$ itself is not a member of the cover. An open cover $\mathcal{U}$ of $X$ is an $\omega$-cover if for each finite $F \subseteq X$, there is $U \in \mathcal{U}$ such that $F \subseteq U$. $\mathcal{U}$ is a $\gamma$-cover of $X$ if it is infinite and for each $x \in X, x$ is a member of all but finitely many members of $\mathcal{U}$. Let $\Omega$ and $\Gamma$ denote the collections of all open $\omega$ covers and $\gamma$-covers of $X$, respectively. Then $\mathrm{U}_{\text {fin }}(\mathrm{O}, \Gamma)$ is the Hurewicz property [28, and $S_{1}(\Omega, \Gamma)$ is the Gerlits-Nagy $\gamma$-property, introduced in the context of function spaces [22]. Additional properties of these types were studied by Arkhangel'skii, Sakai, and others. Some of the properties are relatively new.

The field of selective principles studies the interrelations between all properties defined by the above selective prototypes as well as similar ones, and properties which do not fall into this category but that can be related to properties which do.

In its broadest sense, the field (and even just its problems) cannot be surveyed in a single book chapter. We will restrict attention to its part dealing with sets of real numbers 1 Even there, we omit several important topics. Two of them-topological Ramsey theory and topological game theory - are discussed in Scheepers' chapter.

While all problems we mention are about sets of real numbers, some of them deal with sets of reals not defined by selective principles, and belong to the more classical era of the field. Naturally, we usually mention problems we are more familiar with.

The references we give are usually an accessible account of the problem or related problems, but not necessarily the original source (which is usually cited in the given reference). In fact, most of the problems have been around much before being documented in a publication. Thus, most of the problems posed here should be considered folklore.

The current chapter is a comprehensively revised and updated version of our earlier survey [68].

\section{The Scheepers Diagram Problem}

Each of the properties mentioned in Section 1, where $\mathscr{A}, \mathscr{B}$ range over $\mathrm{O}, \Lambda, \Omega, \Gamma$, is either void or equivalent to one in the following diagram (where an arrow denotes implication) [51, 30].

\footnotetext{
${ }^{1}$ This includes separable zero-dimensional metric spaces, since such spaces are homeomorphic to subsets of the irrational numbers.
} 


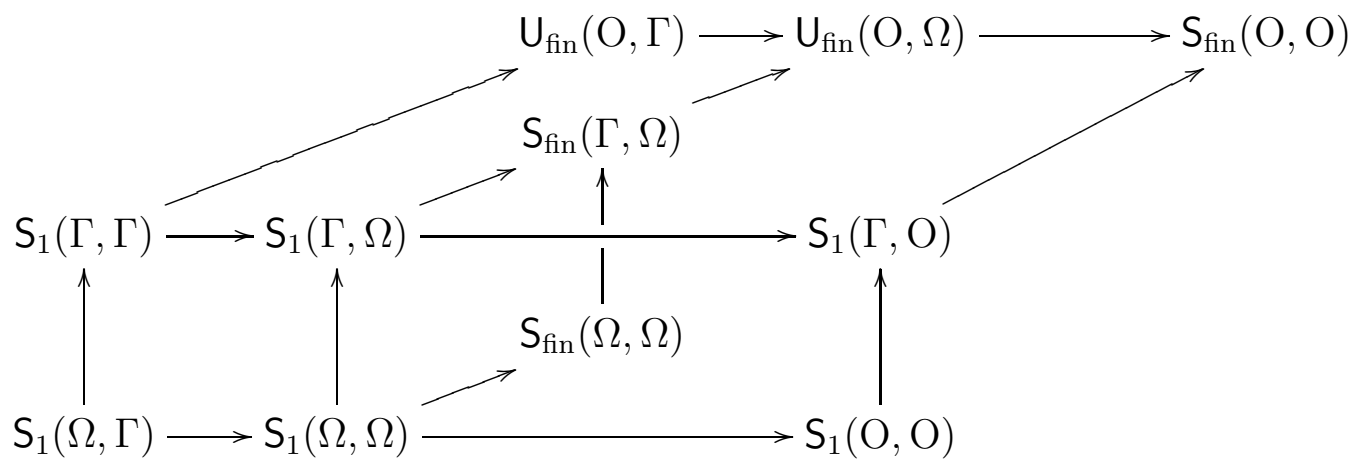

Figure 1. The Scheepers Diagram

Almost all implications which do not appear in Figure 1, and are not compositions of existing implications, are not provable: Assuming the Continuum Hypothesis, there are sets of reals witnessing that [30]. Only the following two implications remain unsettled.

Problem 2.1 ([30]).

(1) Is $\mathrm{U}_{\text {fin }}(\mathrm{O}, \Omega)=\mathrm{S}_{\text {fin }}(\Gamma, \Omega)$ ?

(2) And if not, does $\mathrm{U}_{\text {fin }}(\mathrm{O}, \Gamma)$ imply $\mathrm{S}_{\text {fin }}(\Gamma, \Omega)$ ?

By Borel cover of $X$ we mean a cover of $X$ consisting of Borel subsets of $X$. Let $\mathrm{B}, \mathrm{B}_{\Omega}, \mathrm{B}_{\Gamma}$ denote the collections of countable Borel covers, $\omega$ covers, and $\gamma$-covers of $X$, respectively. Since we are dealing with sets of reals, we may assume that all open covers we consider are countable [64]. It follows that when $\mathscr{A}, \mathscr{B}$ range over $\mathrm{B}, \mathrm{B}_{\Omega}, \mathrm{B}_{\Gamma}$ we get properties stronger than the corresponding ones when $\mathscr{A}, \mathscr{B}$ range over $\mathrm{O}, \Omega, \Gamma$. In the Borel case, more equivalences are known and the following diagram is complete [57].

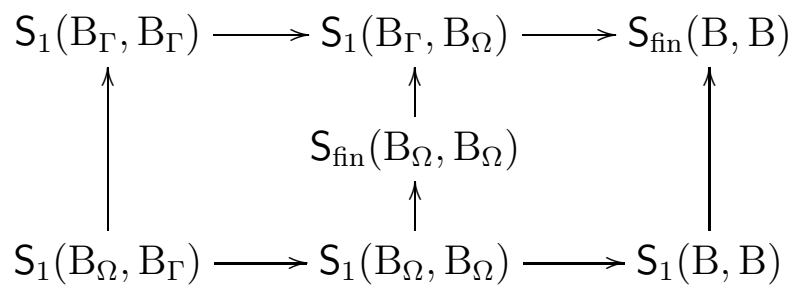

Figure 2. The Scheepers Diagram in the Borel case

In particular, the answer to the Borel counterpart of Problem 2.1] is positive. 
Problem 2.1 can be reformulated in terms of topological properties of sets generating Borel non- $\sigma$-compact groups [81]. This is related to the following problem.

Problem 2.2 ([81]). Can a Borel non- $\sigma$-compact subgroup of a Polish group be generated by a subspace satisfying $\mathrm{U}_{\mathrm{fin}}(\mathrm{O}, \Gamma)$ ?

A set $X \subseteq \mathbb{R}$ satisfies $\mathrm{U}_{\text {fin }}(\mathrm{O}, \Gamma)$ if, and only if, for each $G_{\delta} G \subseteq \mathbb{R}$ containing $X$, there is a $\sigma$-compact $F \subseteq \mathbb{R}$ with $X \subset F \subset G$ [30].

Problem 2.3 ([6, preprint version]). Assume that $X \subseteq B \subseteq \mathbb{R}, B$ is Borel, and $X$ satisfies $\mathrm{U}_{\text {fin }}(\mathrm{O}, \Gamma)$. Must there be a $\sigma$-compact $F$ with $X \subset F \subset B$ ? What if $B$ is $F_{\sigma \delta}$ ?

Update 1. "No", and $\mathfrak{b}=\mathfrak{d}$ suffices for that [6, final version].

The motivation for Problem 2.3 was that a positive answer for its first part would imply a negative answer for Problem 2.2, and a positive answer for its second part would imply a positive answer for Problem $2.1(2)$.

\section{EXAmples Without SPECIAL SET THEORETIC HYPOTHESES}

3.1. Dichotomic examples. Let $\mathcal{J}$ be a property of sets of reals. Sometimes there is a set theoretic hypothesis $P$ independent of ZFC, that can be used to construct an $X \in \mathcal{J}$, and such that its negation $\neg P$ also implies the existence of some $Y \in \mathcal{J}$ (possibly on trivial grounds). In this case, the existence of an $X \in \mathcal{J}$ is a theorem of ZFC.

The hypotheses used in the dichotomic arguments are often related to combinatorial cardinal characteristics of the continuum. See [13] for a survey of these. The critical cardinality of a nontrivial family $\mathcal{J}$ of sets of reals is

$$
\operatorname{non}(\mathcal{J})=\min \{|X|: X \subseteq \mathbb{R} \text { and } X \notin \mathcal{J}\} .
$$

Figure 3 indicates the critical cardinalities of the properties in the Scheepers diagram 1 (we use $\mathcal{M}$ for the ideal of meager sets of reals). The critical cardinalities in the Borel case are equal to those in the open case.

Dichotomic arguments imply the existence (in ZFC) of a set of reals $X$ satisfying $\mathrm{S}_{1}(\Gamma, \Gamma)$ such that $|X|=\mathfrak{t}[55$, and a set of reals satisfying $\mathrm{S}_{\text {fin }}(\Omega, \Omega)$ such that $|X|=\operatorname{cf}(\mathfrak{d})$ [74]. Now, $\operatorname{non}\left(\mathrm{S}_{1}(\Gamma, \Gamma)\right)=\mathfrak{b}$, $\operatorname{non}\left(\mathrm{S}_{\text {fin }}(\Omega, \Omega)\right)=\mathfrak{d}$, and it is consistent that $\mathfrak{b}>\mathfrak{t}$ and $\mathfrak{d}>\operatorname{cf}(\mathfrak{d})$. Thus, these existence results are not satisfactory.

Problem 3.1 ([12]). Does there exist (in ZFC) a set of reals $X$ satisfying $\mathrm{S}_{1}(\Gamma, \Gamma)$ such that $|X|=\mathfrak{b}$ ? 


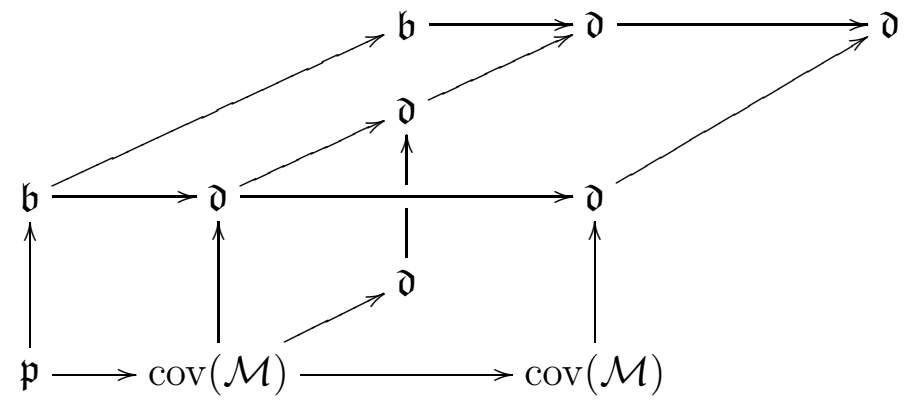

Figure 3. Critical cardinalities in the Scheepers Diagram

Update 2. "No", and the counter-example is Laver's model [38]. The answer becomes "Yes" if we are allowed to pick, instead of one element from each cover, a union of two elements from each cover [71].

Problem 3.2 ([74]). Does there exist (in ZFC) a set of reals satisfying $\mathrm{S}_{\text {fin }}(\Omega, \Omega)$ such that $|X|=\mathfrak{d}$ ?

3.2. Direct constructions. Constructions which do not appeal to a dichotomy are philosophically much more pleasing.

There is a direct construction of a set of reals $H$ satisfying $\mathrm{U}_{\text {fin }}(\mathrm{O}, \Gamma)$ such that $|H|=\mathfrak{b}$ (and such that $H$ does not contain a perfect set) [10]. All finite powers of this set $H$ satisfy $\bigcup_{\text {fin }}(O, \Gamma)$ [12]. In fact, $H$ can be chosen as a subgroup or even a subfield of $\mathbb{R}[65,74]$.

Update 3. A stronger result with simpler proofs is given in [71].

There is also a direct construction of a set of reals $M$ satisfying $\mathrm{S}_{\text {fin }}(\mathrm{O}, \mathrm{O})$ but not $\mathrm{U}_{\text {fin }}(\mathrm{O}, \Gamma)$, such that $|M|=\mathfrak{d}[74$.

Problem 3.3 ([74]). Is there a direct (non-dichotomic) construction of a set of reals $M$ satisfying $\mathrm{S}_{\mathrm{fin}}(\Omega, \Omega)$ but not $\mathrm{U}_{\text {fin }}(\mathrm{O}, \Gamma)$ ?

3.3. The Borel case. Let $\mathcal{J}$ be a property of sets of reals. Borel's Conjecture for $\mathcal{J}$ is the statement "All elements of $\mathcal{J}$ are countable". For all but three of the properties in the Borel case, Borel's Conjecture is consistent.

Problem 3.4 ([45]). Is Borel's Conjecture for $\mathrm{S}_{\mathrm{fin}}(\mathrm{B}, \mathrm{B})$ consistent?

This is the same as asking whether it is consistent that each uncountable set of reals can be mapped onto a dominating subset of $\mathbb{N}^{\mathbb{N}}$ by a Borel function [57]. Problem 3.4 is also open for $\mathrm{S}_{1}\left(\mathrm{~B}_{\Gamma}, \mathrm{B}_{\Omega}\right)$.

Problem 3.5 (Magidor). Is Borel's Conjecture for $\mathrm{S}_{\mathrm{fin}}(\mathrm{B}, \mathrm{B})$ equivalent to Borel's Conjecture for $\mathrm{S}_{1}\left(\mathrm{~B}_{\Gamma}, \mathrm{B}_{\Omega}\right)$ ?

Problem 3.6 ([57]). Is Borel's Conjecture for $\mathrm{S}_{\text {fin }}\left(\mathrm{B}_{\Omega}, \mathrm{B}_{\Omega}\right)$ consistent? 


\section{EXAMPLES FROM CH OR MA}

Consider the Borel case (Figure 2). For each set of reals $X$, we can put "•" in each place in the diagram where the property is satisfied, and "o" elsewhere. There are 14 settings consistent with the arrows in the diagram, and they are all listed in Figure 4.

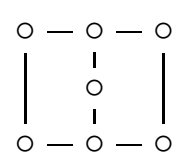

(a)

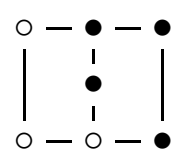

(g)

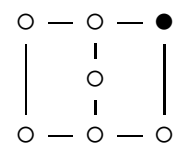

(b)<smiles>[CH]1C[C@H]2OO[C@H]12</smiles>

(h)

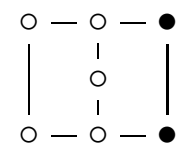

(c)

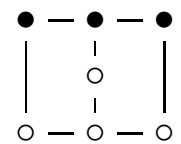

(i)<smiles>C1C[C@H]2OC[C@H]12</smiles>

$(\mathrm{m})$

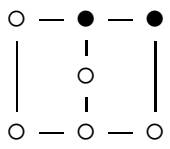

(d)

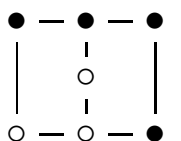

(j)

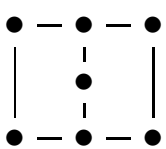

(n)

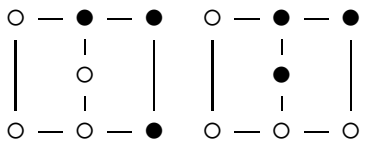

(e)

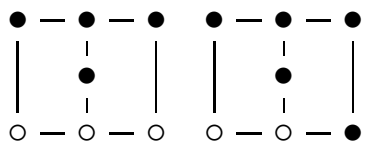

(k)

(l)

Figure 4. The consistent settings

Setting (a) is realized by $\mathbb{R} \backslash \mathbb{Q}$, i.e. $\mathbb{N}^{\mathbb{N}}$.

Assume the Continuum Hypothesis. Settings (c),(h), and (i) were realized in [30], Setting (k) was realized in [65], and Setting (n) was realized in 47, 16, 42. To realize Setting (b), take a set $L$ as in Setting (c) and a set $S$ as in Setting (i), and take $X=L \cup S$. As $\mathrm{S}_{\text {fin }}(\mathrm{B}, \mathrm{B})$ is additive, $X$ satisfies this property. But since $\mathrm{S}_{1}\left(\mathrm{~B}_{\Gamma}, \mathrm{B}_{\Omega}\right)$ and $\mathrm{S}_{1}(\mathrm{~B}, \mathrm{~B})$ are hereditary for subsets [12, $X$ does not satisfy any of these. It seems that using forcing-theoretic arguments similar to those of [16], we can realize Settings (f) and $(\mathrm{m})$.

Problem 4.1. Does the Continuum Hypothesis imply a realization of the settings (d), $(e),(g),(j)$, and $(l)$ ?

All constructions mentioned above can be carried out using Martin's Axiom. Except perhaps Setting (n).

Problem 4.2 ([42]). Does Martin's Axiom imply the existence of a set of reals of cardinality continuum, satisfying $\mathrm{S}_{1}\left(\mathrm{~B}_{\Omega}, \mathrm{B}_{\Gamma}\right)$ ? 


\section{THE $\delta$-PROPERTY}

For a sequence $\left\{X_{n}\right\}_{n \in \mathbb{N}}$ of subsets of $X$, define $\lim \inf X_{n}=\bigcup_{m}$ $\bigcap_{n \geq m} X_{n}$. For a family $\mathcal{F}$ of subsets of $X, L(\mathcal{F})$ denotes its closure under the operation liminf. $X$ has the $\delta$-property [22] if for each open $\omega$-cover $\mathcal{U}$ of $X, X \in L(\mathcal{U})$.

Clearly, $\left(\begin{array}{l}\Omega \\ \Gamma\end{array}\right)$ implies the $\delta$-property. $\mathrm{S}_{1}(\Omega, \Gamma)=\left(\begin{array}{l}\Omega \\ \Gamma\end{array}\right)[22]$.

Problem $5.1([22])$. Is the $\delta$-property equivalent to $\left(\begin{array}{l}\Omega \\ \Gamma\end{array}\right)$ ?

Update 4. "Yes" in the Borel case [44].

Miller points out that, as a union of an increasing sequence of sets with the $\delta$-property has again the $\delta$-property, a negative answer to the following problem implies a negative answer to Problem 5.1.

Problem $5.2([42])$. Does every union of an increasing sequence $\left\{X_{n}\right\}_{n \in \mathbb{N}}$ of sets satisfying $\left(\begin{array}{l}\Omega \\ \Gamma\end{array}\right)$ satisfy $\left(\begin{array}{c}\Omega \\ \Gamma\end{array}\right)$ ?

The answer is positive in the Borel case [65].

Update 5. "Yes" 29]. A simplified proof and applications are given in 43 .

\section{Preservation of properties}

6.1. Heredity. A property of sets of reals is hereditary if for each set of reals $X$ satisfying the property, all subsets of $X$ satisfy that property. None of the selective hypotheses involving open covers is provably hereditary [12]. However, the property $\mathrm{S}_{1}(\mathrm{~B}, \mathrm{~B})$ as well as all properties of the form $\Pi\left(B_{\Gamma}, B\right)$ are hereditary [12] (but $S_{1}\left(B_{\Omega}, B_{\Gamma}\right)$ is not [42]).

Problem 6.1 ([12, 42]). Is $\mathrm{S}_{1}\left(\mathrm{~B}_{\Omega}, \mathrm{B}_{\Omega}\right)$ or $\mathrm{S}_{\text {fin }}\left(\mathrm{B}_{\Omega}, \mathrm{B}_{\Omega}\right)$ hereditary?

Update 6. "Yes" if each countable Borel $\omega$-cover of a set of reals is actually an $\omega$-cover of some Borel superset of that set [43].

All properties in the Scheepers diagram 1, except for the following two, are known to be hereditary for $F_{\sigma}$ subsets [75].

Problem 6.2 ([75]). Are $\mathrm{S}_{\mathrm{fin}}(\Gamma, \Omega)$ and $\mathrm{S}_{1}(\Gamma, \Omega)$ hereditary for $F_{\sigma}$ subsets?

Update 7. "Yes" [43].

The Borel versions of all properties are hereditary for arbitrary Borel subsets [57]. 
6.2. Finite powers. $\mathrm{S}_{1}(\Omega, \Gamma), \mathrm{S}_{1}(\Omega, \Omega)$, and $\mathrm{S}_{\mathrm{fin}}(\Omega, \Omega)$ are the only properties in the open case which are preserved under taking finite powers 30].

Problem $6.3([57])$. Are any of the classes $\mathrm{S}_{1}\left(\mathrm{~B}_{\Omega}, \mathrm{B}_{\Omega}\right)$ or $\mathrm{S}_{\text {fin }}\left(\mathrm{B}_{\Omega}, \mathrm{B}_{\Omega}\right)$ preserved by finite powers?

Assume that $X$ satisfies $\mathrm{S}_{1}\left(\mathrm{~B}_{\Omega}, \mathrm{B}_{\Omega}\right)$ and $Y \subseteq X$. If $\mathrm{S}_{1}\left(\mathrm{~B}_{\Omega}, \mathrm{B}_{\Omega}\right)$ is preserved by finite powers, then $X^{k}$ satisfies $\mathrm{S}_{1}\left(\mathrm{~B}_{\Omega}, \mathrm{B}_{\Omega}\right)$, and in particular $\mathrm{S}_{1}(\mathrm{~B}, \mathrm{~B})$, for all $k$. As $\mathrm{S}_{1}(\mathrm{~B}, \mathrm{~B})$ is hereditary, $Y^{k}$ satisfies $\mathrm{S}_{1}(\mathrm{~B}, \mathrm{~B})$ for all $k$. It follows that $Y$ satisfies $\mathrm{S}_{1}\left(\mathrm{~B}_{\Omega}, \mathrm{B}_{\Omega}\right)$ [57]. Similar assertions for $\mathrm{S}_{\mathrm{fin}}\left(\mathrm{B}_{\Omega}, \mathrm{B}_{\Omega}\right)$ and $\mathrm{S}_{\mathrm{fin}}(\mathrm{B}, \mathrm{B})$ also hold [57]. Thus, a positive answer to Problem 6.3 implies a positive answer to Problem 6.1.

Problem 6.4 ([57]). Is $\mathrm{S}_{1}\left(\mathrm{~B}_{\Omega}, \mathrm{B}_{\Gamma}\right)$ preserved by finite powers?

The corresponding problems for the other classes are settled in the negative [57].

6.3. Products. Some positive results are available for products of sets. E.g., if $X, Y \subseteq \mathbb{R}$ have strong measure zero and $X$ also satisfies $\mathrm{U}_{\text {fin }}(\mathrm{O}, \Gamma)$, then $X \times Y$ has strong measure zero [52].

Problem 6.5 (52]). Assume that $X, Y \subseteq \mathbb{R}$ satisfy $\mathrm{S}_{1}(\mathrm{O}, \mathrm{O})$, and $X$ also satisfies $\mathrm{U}_{\text {fin }}(\mathrm{O}, \Gamma)$. Does it follow that $X \times Y$ satisfies $\mathrm{S}_{1}(\mathrm{O}, \mathrm{O})$ ?

Update 8. "No":

(1) In every extension of a model of the Continuum Hypothesis by $\aleph_{1}$ many Cohen reals, there is a set of reals $X$ satisfying $\mathrm{S}_{1}(\mathrm{O}, \mathrm{O})$ in all finite powers and $\mathrm{U}_{\text {fin }}(\mathrm{O}, \Gamma)$, such that $X^{2}$ does not satisfy $\mathrm{U}_{\text {fin }}(\mathrm{O}, \Gamma)[56$.

(2) The Continuum Hypothesis implies that there are sets of reals $X, Y$ satisfying $\mathrm{S}_{1}(\Omega, \Gamma)$ (which is preserved by finite powers), such that $X \times Y$ does not satisfy $\mathrm{S}_{\text {fin }}(\mathrm{O}, \mathrm{O})$ [39].

It is not even known whether a positive answer follows when $X$ satisfies $S_{1}(\Omega, \Gamma)$.

Update 9. "No", see Update8.

The following problem withstood considerable attacks by several mathematicians. The property in it is equivalent to the Gerlits-Nagy (*) property, and is also equivalent to $\mathrm{S}_{1}\left(\Omega, \mathrm{O}^{\gamma-g p}\right)$ [33].

Problem 6.6. Is $\mathrm{U}_{\text {fin }}(\mathrm{O}, \Gamma) \cap \mathrm{S}_{1}(\mathrm{O}, \mathrm{O})$ preserved by finite products?

A positive answer here implies a positive answer to Problem 6.5. It is not even known whether $\mathrm{U}_{\text {fin }}(\mathrm{O}, \Gamma) \cap \mathrm{S}_{1}(\mathrm{O}, \mathrm{O})$ is preserved by finite powers. 
Update 10. "No", see Update 8.

None of the properties in Figure 1 is provably preserved by finite products [54, 57, 11, 64. Borel's conjecture implies a consistently positive answer for $\mathrm{S}_{1}(\mathrm{O}, \mathrm{O})$ and below it.

Problem 6.7 (Scheepers). Are any of the $\mathrm{S}_{\text {fin }}$ or $\mathrm{U}_{\text {fin }}$ type properties in the Scheepers diagram 1 consistently preserved by finite products?

A natural place to check Problem 6.7 for $\mathrm{S}_{\text {fin }}(\mathrm{O}, \mathrm{O})$ is Miller's model (in which, by the way, $\mathrm{U}_{\text {fin }}(\mathrm{O}, \Omega)=\mathrm{S}_{\text {fin }}(\mathrm{O}, \mathrm{O})[79,[75])$.

Assume that $Y$ has Hausdorff dimension zero. The assumption that $X$ satisfies $\mathrm{S}_{1}(\Omega, \Gamma)$ does not imply that $X \times Y$ has Hausdorff dimension zero. However, if $X$ satisfies $\mathrm{S}_{1}\left(\left\{\mathrm{O}_{n}\right\}_{n \in \mathbb{N}}, \Gamma\right) \cdot 2$ then $X \times Y$ has Hausdorff dimension zero [78].

Problem $6.8([78])$. Assume that $|X|<\mathfrak{p}$. Is it true that for each $Y$ with Hausdorff dimension zero, $X \times Y$ has Hausdorff dimension zero?

Problem 6.9 (Krawczyk). Is it consistent (relative to ZFC) that there are uncountable sets of reals satisfying $\mathrm{S}_{1}(\Omega, \Gamma)$, but for each such set $X$ and each set $Y$ with Hausdorff dimension zero, $X \times Y$ has Hausdorff dimension zero?

6.4. Unions. The question of which of the properties in Figure 1 is provably preserved under taking finite or countable unions (i.e., is additive or $\sigma$-additive) is completely settled. Some of the classes which are not provably additive are consistently additive [70].

Problem $6.10([70])$. Is $\mathrm{S}_{\mathrm{fin}}(\Omega, \Omega)$ consistently additive?

The problem is also open for $S_{1}(\Gamma, \Omega)$ and $S_{\text {fin }}(\Gamma, \Omega)$.

Problem 6.11 ([70]). Is $\mathrm{S}_{\mathrm{fin}}\left(\mathrm{B}_{\Omega}, \mathrm{B}_{\Omega}\right)$ consistently additive?

In some cases, there remains the task to determine the exact additivity number. The additivity number of a nontrivial family $\mathcal{J}$ of sets of reals is

$$
\operatorname{add}(\mathcal{J})=\min \{|\mathcal{F}|: \mathcal{F} \subseteq \mathcal{I} \text { and } \cup \mathcal{F} \notin \mathcal{J}\}
$$

$\max \{\mathfrak{b}, \mathfrak{g}\} \leq \operatorname{add}\left(\mathrm{S}_{\text {fin }}(\mathrm{O}, \mathrm{O})\right) \leq \operatorname{cf}(\mathfrak{d}), \mathfrak{h} \leq \operatorname{add}\left(\mathrm{S}_{1}(\Gamma, \Gamma)\right) \leq \mathfrak{b}$, and $\operatorname{add}(\mathcal{N}) \leq \operatorname{add}\left(\mathrm{S}_{1}(\mathrm{O}, \mathrm{O})\right)[70]$.

Problem 6.12 ([70]). Is $\operatorname{add}\left(\mathrm{S}_{\mathrm{fin}}(\mathrm{O}, \mathrm{O})\right)=\max \{\mathfrak{b}, \mathfrak{g}\}$ ?

\footnotetext{
${ }^{2} \mathrm{~S}_{1}\left(\left\{\mathrm{O}_{n}\right\}_{n \in \mathbb{N}}, \Gamma\right)$ is the strong $\gamma$-property [21, 66]: For each sequence $\left\{\mathcal{U}_{n}\right\}_{n \in \mathbb{N}}$ where for each $n, \mathcal{U}_{n}$ is an open $n$-cover of $X$ (i.e., each $F \subseteq X$ with $|F| \leq n$ is contained in some member of $\left.\mathcal{U}_{n}\right)$, there are $U_{n} \in \mathcal{U}_{n}, n \in \mathbb{N}$, such that $\left\{\mathcal{U}_{n}: n \in \mathbb{N}\right\}$ is a $\gamma$-cover of $X$.
} 
Problem 6.13 ([53]). Is add $\left(\mathrm{S}_{1}(\Gamma, \Gamma)\right)=\mathfrak{b}$ ?

Problem 6.13 is related to Problem 9.1 below. The answer for the Borel version of Problem 6.13 is positive.

Update 11. Consistently, "Yes" [38].

Problem 6.14 ([9]). Is it consistent that $\operatorname{add}(\mathcal{N})<\operatorname{add}\left(\mathrm{S}_{1}(\mathrm{O}, \mathrm{O})\right)$ ?

Another type of problem is exemplified by the following problem. It is easy to see that if $X$ satisfies $\mathrm{S}_{1}(\Omega, \Gamma)$ and $D$ is countable, then $X \cup D$ satisfies $\mathrm{S}_{1}(\Omega, \Gamma)$.

Problem 6.15 (Miller, Tsaban). Assume that $X$ satisfies $\mathrm{S}_{1}(\Omega, \Gamma)$ and $|D|<\mathfrak{p}$. Does $X \cup D$ satisfy $\mathrm{S}_{1}(\Omega, \Gamma)$ ? Is it true under Martin's Axiom when $|D|=\aleph_{1}$ ?

Recently, Francis Jordan proved that for each $D$, the following are equivalent:

(1) $X \cup D$ satisfies $\mathrm{S}_{1}(\Omega, \Gamma)$ for each $X$ satisfying $\mathrm{S}_{1}(\Omega, \Gamma)$;

(2) $X \times D$ satisfies $\mathrm{S}_{1}(\Omega, \Gamma)$ for each $X$ satisfying $\mathrm{S}_{1}(\Omega, \Gamma)$.

\section{MODERN TYPES OF COVERS}

7.1. $\tau$-covers. Recall that by "cover of $X$ " we mean one not containing $X$ as an element. $\mathcal{U}$ is a -cover of $X$ if each $x \in X$ is covered by infinitely many members of $\mathcal{U}$. It is a $\tau$-cover of $X$ if, in addition, for each $x, y \in X$, either $\{U \in \mathcal{U}: x \in U, y \notin U\}$ is finite, or else $\{U \in \mathcal{U}: y \in U, x \notin U\}$ is finite [61]. Let $\mathrm{T}$ denote the collection of open $\tau$-covers of $X$. Then $\Gamma \subseteq \mathrm{T} \subseteq \Omega$.

The most important problem concerning $\tau$-covers is the following.

Problem $7.1([62])$. Is $\left(\begin{array}{c}\Omega \\ \Gamma\end{array}\right)=\left(\begin{array}{c}\Omega \\ \mathrm{T}\end{array}\right)$ ?

This problem is related to many problems posed in [61, 63, 64, 66, 78, 42], etc. The best known result in this direction is that $\left(\begin{array}{l}\Omega \\ \mathrm{T}\end{array}\right)$ implies $\mathrm{S}_{\text {fin }}(\Gamma, \mathrm{T}) 63$.

To state a modest form of Problem [.1, note that if $\left(\begin{array}{c}\Omega \\ \mathrm{T}\end{array}\right)$ implies $\mathrm{S}_{\text {fin }}(\mathrm{T}, \Omega)$, then $\left(\begin{array}{c}\Omega \\ \mathrm{T}\end{array}\right)=\mathrm{S}_{\text {fin }}(\Omega, \mathrm{T})$.

Problem $7.2([63])$. Is $\left(\begin{array}{c}\Omega \\ \mathrm{T}\end{array}\right)=\mathrm{S}_{\mathrm{fin}}(\Omega, \mathrm{T})$ ?

Problem 7.3 (Scheepers). Does $\mathrm{S}_{1}(\Omega, \mathrm{T})$ imply $\mathrm{U}_{\text {fin }}(\mathrm{O}, \Gamma)$ ?

There are many more problems of this type, and they are summarized in [37. 
Not much is known about the preservation of the new properties under set theoretic operations. Miller [42] proved that assuming the Continuum Hypothesis, there exists a set of reals $X$ satisfying $\mathrm{S}_{1}\left(\mathrm{~B}_{\Omega}, \mathrm{B}_{\Gamma}\right)$ and a subset $Y$ of $X$ such that $Y$ does not satisfy $\left(\begin{array}{c}\Omega \\ \mathrm{T}\end{array}\right)$. Together with the remarks preceding Problem 6.1, we have that the only classes (in addition to those in Problem 6.1) for which the heredity problem is not settled are the following ones.

Problem $7.4([12])$. Are any of the properties $\mathrm{S}_{1}\left(\mathrm{~B}_{\mathrm{T}}, \mathrm{B}_{\Gamma}\right), \mathrm{S}_{1}\left(\mathrm{~B}_{\mathrm{T}}, \mathrm{B}_{\mathrm{T}}\right)$, $\mathrm{S}_{1}\left(\mathrm{~B}_{\mathrm{T}}, \mathrm{B}_{\Omega}\right), \mathrm{S}_{1}\left(\mathrm{~B}_{\mathrm{T}}, \mathrm{B}\right), \mathrm{S}_{\mathrm{fin}}\left(\mathrm{B}_{\mathrm{T}}, \mathrm{B}_{\mathrm{T}}\right)$, or $\mathrm{S}_{\mathrm{fin}}\left(\mathrm{B}_{\mathrm{T}}, \mathrm{B}_{\Omega}\right)$, hereditary?

Here are the open problems regarding unions.

Problem 7.5 ([70]). Are any of the properties $\mathrm{S}_{1}(\mathrm{~T}, \mathrm{~T}), \mathrm{S}_{\mathrm{fin}}(\mathrm{T}, \mathrm{T})$, $\mathrm{S}_{1}(\Gamma, \mathrm{T}), \mathrm{S}_{\mathrm{fin}}(\Gamma, \mathrm{T})$, and $\mathrm{U}_{\mathrm{fin}}(\mathrm{O}, \mathrm{T})$ (or any of their Borel versions) additive?

It is consistent that $\mathrm{U}_{\text {fin }}(\mathrm{O}, \Gamma)=\mathrm{U}_{\text {fin }}(\mathrm{O}, \mathrm{T})$, and therefore $\mathrm{U}_{\text {fin }}(\mathrm{O}, \mathrm{T})$ is consistently $\sigma$-additive [80]. $\mathrm{S}_{1}(\mathrm{~T}, \mathrm{~T})$ is preserved under taking finite unions if, and only if, $\mathrm{S}_{1}(\mathrm{~T}, \mathrm{~T})=\mathrm{S}_{1}(\mathrm{~T}, \Gamma)$ [37.

Here are the open problems regarding powers.

Problem 7.6. Are any of the properties

(1) $\mathrm{S}_{1}(\Omega, \mathrm{T})$, or $\mathrm{S}_{\text {fin }}(\Omega, \mathrm{T})$,

(2) $\mathrm{S}_{1}(\mathrm{~T}, \Gamma), \mathrm{S}_{1}(\mathrm{~T}, \mathrm{~T}), \mathrm{S}_{1}(\mathrm{~T}, \Omega), \mathrm{S}_{\mathrm{fin}}(\mathrm{T}, \mathrm{T})$, or $\mathrm{S}_{\mathrm{fin}}(\mathrm{T}, \Omega)$,

preserved under taking finite powers?

Most of these problems are related to Problem 7.1.

A solution to any of the problems involving $\tau$-covers must use new ideas, since this family of covers is not as amenable as the classical ones. In 63] it is shown that if we use an amenable variant of $\tau$-covers (called $\tau^{*}$-covers, see below), then most of the corresponding problems can be solved.

7.2. $\tau^{*}$-covers. $Y \subseteq[\mathbb{N}]^{\aleph_{0}}$ is linearly refinable if for each $y \in Y$ there exists an infinite subset $\hat{y} \subseteq y$ such that the family $\hat{Y}=\{\hat{y}: y \in Y\}$ is linearly (quasi)ordered by $\subseteq^{*}$. A cover $\mathcal{U}=\left\{U_{n}: n \in \mathbb{N}\right\}$ of $X$ is a $\tau^{*}$-cover of $X$ if it is large, and the family of all sets $\left\{n: x \in U_{n}\right\}$, $x \in X$, is linearly refinable. $\mathrm{T}^{*}$ is the collection of all countable open $\tau^{*}$-covers of $X$.

Every analytic space satisfies $\left(\begin{array}{l}\mathrm{T} \\ \Gamma\end{array}\right)[61$.

Problem $7.7([63])$. Does $\{0,1\}^{\mathbb{N}}$ satisfy $\left(\begin{array}{c}\mathrm{T}^{*} \\ \Gamma\end{array}\right)$ ? 
7.3. Glueable covers. Glueability notions for covers appear naturally, under various names, in the studies of selective principles [32, 33, 2, 67.

A cover $\mathcal{U}$ of $X$ is multifinite if there exists a partition of $\mathcal{U}$ into infinitely many finite covers of $X$.

Definition 7.8 (The Gimel operator on families of covers). Let $\mathscr{A}$ be a family of covers of $X . \mathcal{I}(\mathscr{A})$ is the family of all covers $\mathcal{U}$ of $X$ such that: Either $\mathcal{U}$ is multifinite, or there exists a partition $\mathcal{P}$ of $\mathcal{U}$ into finite sets such that $\{\bigcup \mathcal{F}: \mathcal{F} \in \mathcal{P}\} \backslash\{X\} \in \mathscr{A}$.

For each $\mathscr{A}, \mathscr{A} \subseteq I(\mathscr{A})$. An element of $I(\mathscr{A})$ will be called $\mathscr{A}$ glueable. This explains our choice of the Hebrew letter Gimel (I).

$\mathrm{I}(\Gamma) \subseteq \mathrm{I}(\mathrm{T}) \subseteq \mathrm{I}(\Omega) . \quad \mathrm{S}_{\mathrm{fin}}(\mathrm{O}, \mathrm{O})=\mathrm{S}_{\text {fin }}(\Omega, \mathrm{O})$ [51], $\mathrm{U}_{\text {fin }}(\mathrm{O}, \Gamma)=$ $\mathrm{S}_{\text {fin }}(\Omega, \beth(\Gamma))$ [33], and $\mathrm{U}_{\text {fin }}(\mathrm{O}, \Omega)=\mathrm{S}_{\text {fin }}(\Omega, I(\Omega))$ [2]. These results are generalized in [?]. A positive answer to the following problem is consistent [80].

Problem 7.9. Is $\mathrm{U}_{\text {fin }}(\mathrm{O}, \mathrm{T})=\mathrm{S}_{\text {fin }}(\Omega, \beth(\mathrm{T}))$ ?

Update 12. All results mentioned before the last problem are generalized in [?]. In particular, $\mathrm{U}_{\text {fin }}\left(\mathrm{O}, \mathrm{T}^{*}\right)=\mathrm{S}_{\text {fin }}\left(\Omega, \beth\left(\mathrm{T}^{*}\right)\right)$. However, the original problem remains open.

$\mathrm{S}_{1}(\Omega, I(\Omega))$ is strictly stronger than $\mathrm{S}_{1}(\mathrm{O}, \mathrm{O})[66] . \quad \mathrm{S}_{1}(\Omega, I(\Omega))=$ $\mathrm{U}_{\text {fin }}(\mathrm{O}, \Omega) \cap \mathrm{S}_{1}(\mathrm{O}, \mathrm{O})[66$, so the following problem can also be stated in classical terms.

Problem $7.10([2])$. Is $\mathrm{S}_{1}(\Omega, \Omega)=\mathrm{S}_{1}(\Omega, \beth(\Omega))$ ?

Update 13. $\mathrm{S}_{1}(\Omega, \beth(\Omega))=\mathrm{S}_{1}(\mathrm{O}, \mathrm{O}) \cap \mathrm{U}_{\text {fin }}(\mathrm{O}, \Omega)[?]$. The problem, however, is still open.

$\mathrm{U}_{\text {fin }}(\mathrm{O}, \Gamma)=\left(\begin{array}{c}\Lambda \\ \mathrm{I}(\Gamma)\end{array}\right)$ [67]. Zdomskyy proved that a positive answer to the following problem follows from $\mathrm{NCF}$.

Problem 7.11. Is $\mathrm{U}_{\text {fin }}(\mathrm{O}, \Omega)=\left(\begin{array}{c}\Lambda \\ \mathrm{J}(\Omega)\end{array}\right)$ ?

\section{Splittability}

Assume that $\mathscr{A}$ and $\mathscr{B}$ are collections of covers of a space $X$. The following property was introduced in [51], in connection to Ramsey Theory.

$\operatorname{Split}(\mathscr{A}, \mathscr{B})$ : Every cover $\mathcal{U} \in \mathscr{A}$ can be split into two disjoint subcovers $\mathcal{V}$ and $\mathcal{W}$, each containing an elements of $\mathscr{B}$. 
If we consider this prototype with $\mathscr{A}, \mathscr{B} \in\{\Lambda, \Omega, \mathrm{T}, \Gamma\}$, we obtain 16 properties, each of which being either trivial or equivalent to one in Figure 5. In this diagram, the dotted implications are open. The implication (1) in this diagram holds if, and only if, its implication (2) holds, and if (1) (and (2)) holds, then (3) holds, either.

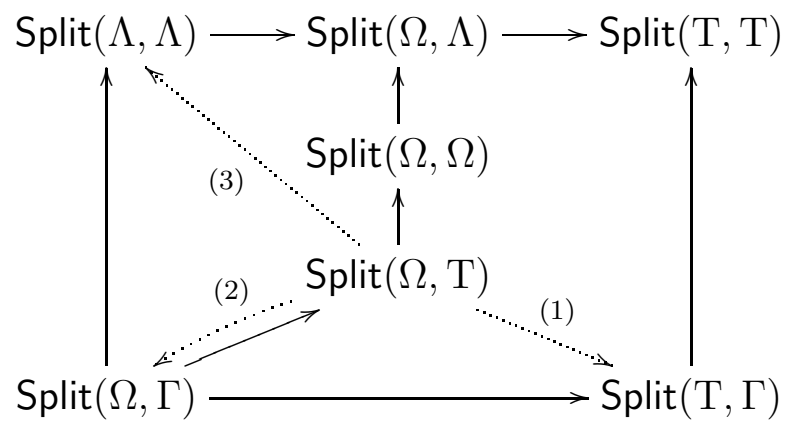

FigURE 5.

Problem 8.1 ([64]).

(1) Does Split $(\Omega, \mathrm{T})$ imply Split $(\mathrm{T}, \Gamma)$ ?

(2) And if not, then does $\operatorname{Split}(\Omega, \mathrm{T})$ imply $\operatorname{Split}(\Lambda, \Lambda)$ ?

The product of a $\sigma$-compact $X$ with $Y$ satisfying $\mathrm{U}_{\text {fin }}(\mathrm{O}, \mathscr{B})(\mathscr{B} \in$ $\{\mathrm{O}, \Omega, \Gamma\})$ satisfies $\mathrm{U}_{\text {fin }}(\mathrm{O}, \mathscr{B})[75,70]$.

Problem 8.2 (Zdomskyy). Assume that $X$ is compact and $Y$ satisfies Split $(\Lambda, \Lambda)$. Does $X \times Y$ satisfy $\operatorname{Split}(\Lambda, \Lambda)$ ?

Problem 8.2 is also open for the other splitting properties.

Problem 8.3 ([70]). Improve the lower bound or the upper bound in the inequality $\aleph_{1} \leq \operatorname{add}(\operatorname{Split}(\Omega, \Lambda)) \leq \mathfrak{c}$.

Problem 8.4 ([70]). Can the lower bound $\mathfrak{u}$ on $\operatorname{add}(\operatorname{Split}(\mathrm{T}, \mathrm{T})$ ) be improved?

All problems below are settled for the properties which do not appear in them.

Problem 8.5 ([64]). Is Split $(\Lambda, \Lambda)$ additive?

Split $(\Lambda, \Lambda)$ is consistently additive [79, 70].

Problem 8.6 ([64]). Are any of the properties $\operatorname{Split}\left(\mathrm{B}_{\Omega}, \mathrm{B}_{\Lambda}\right)$, Split $\left(\mathrm{B}_{\Omega}, \mathrm{B}_{\Omega}\right)$, Split $\left(\mathrm{B}_{\mathrm{T}}, \mathrm{B}_{\mathrm{T}}\right)$, and $\operatorname{Split}\left(\mathrm{B}_{\mathrm{T}}, \mathrm{B}_{\Gamma}\right)$ hereditary?

Problem 8.7 ([64]). Are any of the properties Split $(\Omega, \Omega)$, Split $(\Omega, T)$, or Split $(\mathrm{T}, \mathrm{T})$ preserved under taking finite powers? 


\section{FunCtion SPACES AND LOCAL-GLOBAL PRINCIPLES}

Let $X$ be a topological space, and $x \in X$. A subset $A$ of $X$ converges to $x, x=\lim A$, if $A$ is infinite, $x \notin A$, and for each neighborhood $U$ of $x, A \backslash U$ is finite. Consider the following collections:

$$
\begin{aligned}
\Omega_{x} & =\{A \subseteq X: x \in \bar{A} \backslash A\} \\
\Gamma_{x} & =\left\{A \subseteq X:|A|=\aleph_{0} \text { and } x=\lim A\right\}
\end{aligned}
$$

$\Gamma_{x} \subseteq \Omega_{x}$. The following implications hold, and none further [4].

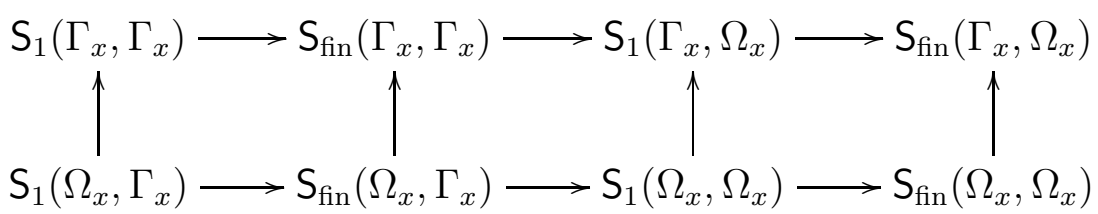

In the current section, when we write $\Pi\left(\mathscr{A}_{x}, \mathscr{B}_{x}\right)$ without specifying $x$, we mean $(\forall x) \Pi\left(\mathscr{A}_{x}, \mathscr{B}_{x}\right) . \mathrm{S}_{\text {fin }}\left(\Omega_{x}, \Omega_{x}\right)$ is Arkhangel'skii's countable fan tightness, and $\mathrm{S}_{1}\left(\Omega_{x}, \Omega_{x}\right)$ is Sakai's countable strong fan tightness. $\mathrm{S}_{1}\left(\Gamma_{x}, \Gamma_{x}\right)$ and $\mathrm{S}_{\mathrm{fin}}\left(\Gamma_{x}, \Gamma_{x}\right)$ are Arkhangel'skii's properties $\alpha_{2}$ and $\alpha_{4}$, respectively.

In the remainder of this section, $X$ will always denote a subset of $\mathbb{R} \backslash \mathbb{Q}$. The set of all real-valued functions on $X$, denoted $\mathbb{R}^{X}$, is equipped with the Tychonoff product topology. $C_{p}(X)$ is the subspace of $\mathbb{R}^{X}$ consisting of the continuous real-valued functions on $X$. The topology of $C_{p}(X)$ is known as the topology of pointwise convergence. The constant zero element of $C_{p}(X)$ is denoted $\mathbf{0}$.

For some of the pairs $(\mathscr{A}, \mathscr{B}) \in\{\Omega, \Gamma\}^{2}$ and $\Pi \in\left\{\mathrm{S}_{1}, \mathrm{~S}_{\mathrm{fin}}\right\}$, it is known that $C_{p}(X)$ satisfies $\Pi\left(\mathscr{A}_{\mathbf{0}}, \mathscr{B}_{\mathbf{0}}\right)$ if, and only if, $X$ satisfies $\Pi(\mathscr{A}, \mathscr{B})$ (see [58] for a summary).

Fremlin's $s_{1}$ for $X$ and Bukovský's wQN for $X$ are equivalent to $\mathrm{S}_{1}\left(\Gamma_{\mathbf{0}}, \Gamma_{\mathbf{0}}\right)$ for $C_{p}(X)$ [53, 20]. In a manner similar to the observation made in Section 3 of [53], a positive solution to Problem 6.13 should imply a positive solution to the following problem.

Problem 9.1 ([19]). Assume that $\kappa<\mathfrak{b}$, and for each $\alpha<\lambda, C_{p}\left(X_{\alpha}\right)$ satisfies $\mathrm{S}_{1}\left(\Gamma_{\mathbf{0}}, \Gamma_{\mathbf{0}}\right)$. Does $C_{p}\left(\bigcup_{\alpha<\kappa} X_{\alpha}\right)$ satisfy $\mathrm{S}_{1}\left(\Gamma_{\mathbf{0}}, \Gamma_{\mathbf{0}}\right)$ ?

Update 14. Consistently, "Yes" 38].

If $X$ satisfies $\mathrm{S}_{1}(\Gamma, \Gamma)$, then $C_{p}(X)$ satisfies $\mathrm{S}_{1}\left(\Gamma_{\mathbf{0}}, \Gamma_{\mathbf{0}}\right)[53]$.

Problem $9.2([53])$. Is $\mathrm{S}_{1}\left(\Gamma_{\mathbf{0}}, \Gamma_{\mathbf{0}}\right)$ for $C_{p}(X)$ equivalent to $\mathrm{S}_{1}(\Gamma, \Gamma)$ for $X$ ?

If the answer is positive, then Problems 6.13 and 9.1 coincide. There are several partial solutions to Problem 9.2: First, for each $Y \subseteq X$ 
$C_{p}(X)$ is $\mathrm{S}_{1}\left(\Gamma_{\mathbf{0}}, \Gamma_{\mathbf{0}}\right)$, then $X$ satisfies $\mathrm{S}_{1}(\Gamma, \Gamma)$ [24. Second, $\mathrm{S}_{1}\left(\Gamma_{\mathbf{0}}, \Gamma_{\mathbf{0}}\right)$ for $C_{p}(X)$ is equivalent to $\overline{\mathrm{S}}_{1}(\Gamma, \Gamma)$ for $X$, where $\overline{\mathrm{S}}_{1}$ is like $\mathrm{S}_{1}$ with the following additional restriction on the given $\gamma$-covers $\mathcal{U}_{n}$ : For each $n$, the family of closures of the elements of $\mathcal{U}_{n+1}$ refines $\mathcal{U}_{n}$ [17]. Finally, $\mathrm{S}_{1}\left(\Gamma_{\mathbf{0}}, \Gamma_{\mathbf{0}}\right)$ for $C_{p}(X)$ is equivalent to $\mathrm{S}_{1}\left(C_{\Gamma}, C_{\Gamma}\right)$ for $X$, where $C_{\Gamma}$ is the collection of clopen $\gamma$-covers of $X$ [50]. This reduces Problem 9.2 to the question whether $\mathrm{S}_{1}(\Gamma, \Gamma)=\mathrm{S}_{1}\left(C_{\Gamma}, C_{\Gamma}\right)$.

The following also seems to be open.

Problem 9.3 (Scheepers). Is $\mathrm{S}_{1}\left(\Gamma_{\mathbf{0}}, \Omega_{\mathbf{0}}\right)$ for $C_{p}(X)$ equivalent to $\mathrm{S}_{1}(\Gamma, \Omega)$ for $X$ ?

$\mathrm{S}_{1}\left(\Gamma_{\mathbf{0}}, \Omega_{\mathbf{0}}\right)$ for $C_{p}(X)$ is equivalent to $\mathrm{S}_{1}\left(C_{\Gamma}, C_{\Omega}\right)$ for $X$, where $C_{\Omega}$ is the collection of clopen $\omega$-covers of $X$ [50], so we really want to know whether $\mathrm{S}_{1}(\Gamma, \Omega)=\mathrm{S}_{1}\left(C_{\Gamma}, C_{\Omega}\right)$.

A topological space $Y$ is $\kappa$-Fréchet if it satisfies $\left(\begin{array}{c}O\left(\Omega_{x}\right) \\ \Gamma_{x}\end{array}\right)$, where $O\left(\Omega_{x}\right)$ is the family of elements of $\Omega_{x}$ which are open.

Problem 9.4 ([49]). What is the minimal cardinality of a set $X \subseteq \mathbb{R}$ such that $C_{p}(X)$ does not satisfy $\left(\begin{array}{c}O\left(\Omega_{x}\right) \\ \Gamma_{x}\end{array}\right)$ ?

The answer is at least $\mathfrak{b}[49$.

There are many additional important questions about these and related kinds of local-global principles. Some of them are surveyed in [23].

\section{TOPOLOGICAL GROUPS}

Let $\mathrm{O}_{\text {nbd }}$ denote the covers of $G$ of the form $\{g \cdot U: g \in G\}$, where $U$ is a neighborhood of the unit element of $G$. Okunev has introduced the property $\mathrm{S}_{\text {fin }}\left(\mathrm{O}_{\text {nbd }}, \mathrm{O}\right)$, traditionally called o-boundedness or Menger-boundedness. Let $\Omega_{\mathrm{nbd}}$ denote the covers of $G$ of the form $\left\{F \cdot U: F \in[G]^{<\aleph_{0}}\right\}$, where $U$ is a neighborhood of the unit element of $G$, such that for each $F \in[G]^{<\aleph_{0}}, F \cdot U \neq G$. Kočinac has introduced $\mathrm{S}_{1}\left(\Omega_{\mathrm{nbd}}, \Omega\right), \mathrm{S}_{1}\left(\Omega_{\mathrm{nbd}}, \Gamma\right)$, and $\mathrm{S}_{1}\left(\mathrm{O}_{\mathrm{nbd}}, \mathrm{O}_{\mathrm{nbd}}\right)$, traditionally called Scheepers-boundedness, Hurewicz-boundedness, and Rothbergerboundedness.

The relations among these boundedness properties and their topological counterparts were studied in many papers, see [25, 26, 35, 65, 5, 3, 1, 81, 36], and references therein. In particular, the following 
diagram of implications is complete.

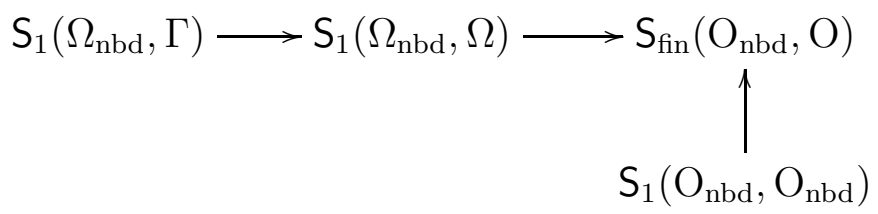

$\mathrm{S}_{\mathrm{fin}}\left(\mathrm{O}_{\mathrm{nbd}}, \mathrm{O}\right)$ is not provably preserved under cartesian products [5, 34, 65.

Problem 10.1 (Tkačenko). Are there, in $Z F C$, groups $G, H$ satisfying $\mathrm{S}_{\mathrm{fin}}\left(\mathrm{O}_{\mathrm{nbd}}, \mathrm{O}\right)$ such that $G \times H$ does not satisfy $\mathrm{S}_{\mathrm{fin}}\left(\mathrm{O}_{\mathrm{nbd}}, \mathrm{O}\right)$ ?

A topological group $G$ satisfies $\mathrm{S}_{1}\left(\Omega_{\mathrm{nbd}}, \Omega\right)$ if, and only if, all finite powers of $G$ satisfy $\mathrm{S}_{\mathrm{fin}}\left(\mathrm{O}_{\mathrm{nbd}}, \mathrm{O}\right)[3]$. Thus, the case where $G=H$ in Problem 10.1 is related to the following problem.

Problem 10.2 ([36]). Is $\mathrm{S}_{1}\left(\Omega_{\mathrm{nbd}}, \Omega\right)=\mathrm{S}_{\mathrm{fin}}\left(\mathrm{O}_{\mathrm{nbd}}, \mathrm{O}\right)$ for separable metrizable groups? Specifically:

(1) Does the Continuum Hypothesis imply the existence of a separable metrizable group $G$ satisfying $\mathrm{S}_{\mathrm{fin}}\left(\mathrm{O}_{\mathrm{nbd}}, \mathrm{O}\right)$ but not $\mathrm{S}_{1}\left(\Omega_{\mathrm{nbd}}\right.$, $\Omega)$ ?

(2) Is it consistent that $\mathrm{S}_{\mathrm{fin}}\left(\mathrm{O}_{\mathrm{nbd}}, \mathrm{O}\right)=\mathrm{S}_{1}\left(\Omega_{\mathrm{nbd}}, \Omega\right)$ for separable metrizable groups?

Update 15. "Yes" for (1): the Continuum Hypothesis implies that for each $k$, there is a subgroup of $\mathbb{Z}^{\mathbb{N}}$ such that $G^{k}$ is $\mathrm{S}_{\mathrm{fin}}\left(\mathrm{O}_{\mathrm{nbd}}, \mathrm{O}\right)$ but $G^{k+1}$ is not [?].

If $G$ is analytic and does not satisfy $\mathrm{S}_{1}\left(\Omega_{\mathrm{nbd}}, \Gamma\right)$, then $G^{2}$ does not satisfy $\mathrm{S}_{\text {fin }}\left(\mathrm{O}_{\mathrm{nbd}}, \mathrm{O}\right)$. Thus, for analytic groups, $\mathrm{S}_{1}\left(\Omega_{\mathrm{nbd}}, \Gamma\right)=\mathrm{S}_{1}\left(\Omega_{\mathrm{nbd}}, \Omega\right)$ [7]. Moreover, for analytic abelian groups, $\mathrm{S}_{\mathrm{fin}}\left(\mathrm{O}_{\mathrm{nbd}}, \mathrm{O}\right)=\mathrm{S}_{1}\left(\Omega_{\mathrm{nbd}}, \Gamma\right)$ [7]. For general analytic groups this is open.

Problem $10.3([7])$. Is there an analytic group satisfying $\mathrm{S}_{\mathrm{fin}}\left(\mathrm{O}_{\mathrm{nbd}}, \mathrm{O}\right)$ but $\operatorname{not} \mathrm{S}_{1}\left(\Omega_{\mathrm{nbd}}, \Gamma\right)$ ?

It seems that $\mathbb{Z}^{\mathbb{N}}$ for boundedness properties of topological groups is like $\mathbb{R}$ for topological and measure theoretic notions of smallness [36]. Thus, unless otherwise indicated, all of the problems in the remainder of this section are concerning subgroups of $\mathbb{Z}^{\mathbb{N}}$.

Say that $G \leq \mathbb{Z}^{\mathbb{N}}$ is bounded if $\{|g|: g \in G\}$ is bounded (with respect to $\leq^{*}$ ), where $|g|$ denotes the absolute value of $g$. For subgroups of $\mathbb{Z}^{\mathbb{N}}$ :

(1) $G$ satisfies $\mathrm{S}_{1}\left(\Omega_{\mathrm{nbd}}, \Gamma\right)$ if, and only if, $G$ is bounded [1].

(2) $G$ satisfies $\mathrm{S}_{1}\left(\mathrm{O}_{\text {nbd }}, \mathrm{O}_{\mathrm{nbd}}\right)$ if, and only if, $G$ has strong measure zero [3]. 
Problem 10.4 ([36]). Is it consistent that there is $G \leq \mathbb{Z}^{\mathbb{N}}$ such that $G$ has strong measure zero, is unbounded, and does not satisfy $\mathrm{S}_{\mathrm{fin}}(\mathrm{O}, \mathrm{O})$ ?

Problem 10.5 ([36]). Is it consistent that there is $G \leq \mathbb{Z}^{\mathbb{N}}$ such that $G$ has strong measure zero and satisfies $\mathrm{S}_{\text {fin }}(\mathrm{O}, \mathrm{O})$, but is unbounded and does not satisfy $\mathrm{S}_{1}(\mathrm{O}, \mathrm{O})$ ?

Update 16. "Yes" for the last two problems [76]. "Yes" under Martin's Axiom [46].

Some open problems involve only the standard covering properties. The following problem is related to Problem 3.2.

Problem 10.6 ([65]). Is there (in $Z F C$ ) a group $G \leq \mathbb{Z}^{\mathbb{N}}$ of cardinality $\mathfrak{d}$ satisfying $\mathrm{S}_{\text {fin }}(\mathrm{O}, \mathrm{O})$ ?

Problem $10.7(65])$. Does the Continuum Hypothesis imply the existence of a a group $G \leq \mathbb{Z}^{\mathbb{N}}$ of cardinality $\mathfrak{c}$ satisfying $\mathrm{S}_{1}\left(\mathrm{~B}_{\Omega}, \mathrm{B}_{\Gamma}\right)$, or at least $\mathrm{S}_{1}(\Omega, \Gamma)$ ?

Update 17. "Yes" for $S_{1}(\Omega, \Gamma)$ [43]. Possibly, a set of Sacks reals in the spirit of [16] would satisfy $\mathrm{S}_{1}\left(\mathrm{~B}_{\Omega}, \mathrm{B}_{\Gamma}\right)$ in all finite powers and thus, by the methods of [43], generate a group satisfying $S_{1}\left(B_{\Omega}, B_{\Gamma}\right)$.

Some approximations to Problem 10.7 are given in [65]: the Continuum Hypothesis implies the existence of groups satisfying $S_{1}\left(B_{\Omega}, B_{\Omega}\right)$ and of groups satisfying $S_{1}\left(B_{\Gamma}, B_{\Gamma}\right)$ in all finite powers. The answer for Problem 10.7 is positive if it is for 5.2. It is also positive for the property $(\delta)$. To get a complete positive answer, it suffices to construct a set $X \subseteq \mathbb{Z}^{\mathbb{N}}$ such that all finite powers of $X$ satisfy $\mathrm{S}_{1}\left(\mathrm{~B}_{\Omega}, \mathrm{B}_{\Gamma}\right)$. Thus, it suffices to have a positive answer for Problem 6.4.

Finally, recall Problem 2.2, and see the other problems in [81].

\section{Cardinal characteristics of the Continuum}

We mention here several problems in the field which are connected to selective principles.

The main open problem in the field is the Minimal Tower Problem. This problem has motivated the study of $\tau$-covers.

Problem 11.1 ([18]). Is it consistent that $\mathfrak{p}<\mathfrak{t}$ ?

Shelah is currently working on a possible positive solution to this problem.

The study of $\tau^{*}$-covers, a variant of $\tau$-covers, led to the following problem. A family $\mathcal{F} \subseteq[\mathbb{N}]^{\aleph_{0}}$ is linearly refinable if for each $A \in \mathcal{F}$ there exists an infinite subset $\hat{A} \subseteq A$ such that the family $\hat{\mathcal{F}}=\{\hat{A}$ : 
$A \in \mathcal{F}\}$ is linearly (quasi)ordered by $\subseteq^{*}$. $\mathfrak{p}^{*}$ is the minimal size of a centered family in $[\mathbb{N}]^{\aleph_{0}}$ which is not linearly refinable.

$$
\mathfrak{p}=\min \left\{\mathfrak{p}^{*}, \mathfrak{t}\right\} \text {, and } \mathfrak{p}^{*} \leq \mathfrak{d} 63 \text {. }
$$

Problem $11.2([63,59])$. Is $\mathfrak{p}=\mathfrak{p}^{*}$ ?

A family $\mathcal{A} \subseteq\left([\mathbb{N}]^{\aleph_{0}}\right)^{\mathbb{N}}$ is a $\tau$-family if for each $n,\{A(n): A \in \mathcal{A}\}$ is linearly ordered by $\subseteq{ }^{*}$. A family $\mathcal{A} \subseteq\left([\mathbb{N}]^{\aleph_{0}}\right)^{\mathbb{N}}$ is o-diagonalizable if there is $g \in \mathbb{N}^{\mathbb{N}}$ such that:

$$
(\forall A \in \mathcal{A})(\exists n) g(n) \in A(n) .
$$

Let $\mathfrak{o} d$ denote the minimal cardinality of a $\tau$-family which is not $o$ diagonalizable. $\operatorname{non}\left(\mathrm{S}_{1}(\mathrm{~T}, \mathrm{O})\right)=\mathfrak{o} \mathfrak{d}$ [37. $\mathfrak{o} \mathfrak{d}$ is the "tower version" of $\operatorname{cov}(\mathcal{M})$ : If we replace "linearly ordered by $\subseteq^{*}$ " by "centered" in the definition of $\mathfrak{o d}$, then we obtain $\operatorname{cov}(\mathcal{M})$. Thus, $\operatorname{cov}(\mathcal{M}) \leq \mathfrak{o d}$. If $\operatorname{cov}(\mathcal{M})=\aleph_{1}$, then $\mathfrak{o d}=\aleph_{1}$ either [37].

Problem 11.3 ([37]). Is it consistent that $\operatorname{cov}(\mathcal{M})<\mathfrak{o d}$ ?

Another variant of the minimal tower problem is the following. For a cardinal number $\kappa>1$ (finite or infinite), define $\mathfrak{p}_{\kappa}$ to be the minimal cardinality of a centered subset of $[\mathbb{N}]^{\aleph_{0}}$ which cannot be partitioned into less than $\kappa$ sets each having a pseudo-intersection.

It is easy to see that $\mathfrak{p}=\mathfrak{p}_{2}=\mathfrak{p}_{3}=\cdots=\mathfrak{p}_{\aleph_{0}}$, and $\mathfrak{p}_{\mathfrak{t}}=\mathfrak{t}$. It turns out that $\mathfrak{p}=\mathfrak{p}_{\aleph_{1}}$ [60]. We get a hierarchy of cardinals between $\mathfrak{p}$ and $\mathfrak{t}$ :

$$
\mathfrak{p}=\mathfrak{p}_{\aleph_{1}} \leq \mathfrak{p}_{\aleph_{2}} \leq \ldots \leq \mathfrak{p}_{\mathfrak{t}}=\mathfrak{t}
$$

Problem 11.4. Is $\mathfrak{p}_{\mathfrak{p}}=\mathfrak{t}$ ?

Finally, consider the following Ramsey-theoretic cardinal: For a subset $Y$ of $\mathbb{N}^{\mathbb{N}}$ and $g \in \mathbb{N}^{\mathbb{N}}$, we say that $g$ avoids middles in $Y$ if:

(1) for each $f \in Y, g \mathbb{Z}^{*} f$;

(2) for all $f, h \in Y$ at least one of the sets $\{n: f(n)<g(n) \leq h(n)\}$ and $\{n: h(n)<g(n) \leq f(n)\}$ is finite.

$\operatorname{add}(\mathfrak{X}, \mathfrak{D})$ is the minimal cardinality $\kappa$ of a dominating $Y \subseteq \mathbb{N}^{\mathbb{N}}$ such that for each partition of $Y$ into $\kappa$ many pieces, there is a piece such that no $g$ avoids middles in that piece. This cardinal is studied in [59].

Problem $11.5([59])$. Is $\operatorname{cov}(\mathcal{M}) \leq \operatorname{add}(\mathfrak{X}, \mathfrak{D})$ ?

\section{Additional PRoblems and other special Sets of Reals}

If a set of reals $X$ satisfies $\mathrm{S}_{\text {fin }}(\mathrm{O}, \mathrm{O})$, then for each continuous image $Y$ of $X$ in $\mathbb{N}^{\mathbb{N}}, Y$ is not dominating, that is, the set $G=\left\{g \in \mathbb{N}^{\mathbb{N}}\right.$ : $\left.(\exists f \in Y) g \leq^{*} f\right\}$ is not equal to $\mathbb{N}^{\mathbb{N}}[27]$. In fact, $G$ satisfies $\mathrm{S}_{\mathrm{fin}}(\mathrm{O}, \mathrm{O})$ [73]. 
If $X$ satisfies $\mathrm{U}_{\text {fin }}(\mathrm{O}, \Omega)$, then for each continuous image $Y$ of $X$ in $\mathbb{N}^{\mathbb{N}},\left\{g \in \mathbb{N}^{\mathbb{N}}:(\exists k)\left(\exists f_{1}, \ldots, f_{k} \in Y\right) g \leq^{*} \max \left\{f_{1}, \ldots, f_{k}\right\}\right\}$ is not comeager [75].

Problem 12.1 (75]). Assume that $X$ satisfies $\mathrm{U}_{\text {fin }}(\mathrm{O}, \Omega)$. Does it follow that

$$
G=\left\{g \in \mathbb{N}^{\mathbb{N}}:(\exists k)\left(\exists f_{1}, \ldots, f_{k} \in Y\right) g \leq^{*} \max \left\{f_{1}, \ldots, f_{k}\right\}\right\}
$$

satisfies $\mathrm{U}_{\mathrm{fin}}(\mathrm{O}, \Omega)$ ?

We now give a short selection of problems on special sets of reals. See [41] or the cited references for the definitions.

$X \subseteq \mathbb{R}$ is a $\nu$-set if for each $Y \subseteq X$ which is nowhere dense in $X$, $Y$ is countable (i.e., $X$ is Luzin relative to itself). Every continuous image of a $\nu$-set has the property assumed in the following problem.

Problem 12.2 ([15]). Assume that $X \subseteq \mathbb{R}$, and for each $Y \subseteq X, Y$ is concentrated on a countable subset of $Y$. Does it follow that $X$ is a continuous image of a $\nu$-set?

Problem $12.3([8])$. Is it consistent that $\operatorname{cov}(\mathcal{M})=\aleph_{1}<\mathfrak{c}=\aleph_{\omega_{1}}$, and there is a $\mathfrak{c}$-Luzin set (i.e., $L$ with $|L|=\mathfrak{c}$ and $|L \cap M|<\mathfrak{c}$ for all meager $M \subseteq \mathbb{R})$ ?

Problem 12.4 ([8]). Assume that every strong measure zero set of reals is meager-additive. Does Borel's Conjecture follow?

The assumption in the last problem implies that $\operatorname{cov}(\mathcal{M})=$ non $(S M Z)<\operatorname{cof}(\mathcal{M})$.

If $X, Y \subseteq\{0,1\}^{\mathbb{N}}$ are meager-additive, then $X \times Y$ is a meageradditive subset of $\{0,1\}^{\mathbb{N}} \times\{0,1\}^{\mathbb{N}}$. The same is true for null-additive subsets of $\{0,1\}^{\mathbb{N}}$. For the real line this is open.

Problem 12.5 ([72]). Assume that $X, Y \subseteq \mathbb{R}$ are meager- (respectively, null-) additive. Does it follow that $X \times Y$ is meager-(respectively, null-) additive?

Update 18. "Yes" for meager-additive [77].

Weiss proved that every meager-additive subset of the Cantor space, when viewed as a subset of $\mathbb{R}$ (where each $f \in\{0,1\}^{\mathbb{N}}$ is identified with $\sum_{n} f(n) / 2^{n}$ ), is meager-additive (with respect to the usual addition in $\mathbb{R})$; and similarly for null-additive.

Problem 12.6 ([72]). Assume that $X \subseteq \mathbb{R}$ is meager- (respectively, null-) additive, and $X \subseteq[0,1]$. Does it follow that $X$ is meager- (respectively, null-) additive when viewed as a subset of $\{0,1\}^{\mathbb{N}}$ ? 
Update 19. "Yes" for meager-additive [77].

For a set $H$, define $H_{x}=\{y:(x, y) \in H\}$.

Problem 12.7 (Bartoszyński). Assume that $X \subseteq \mathbb{N}^{\mathbb{N}}$ is nonmeager and $Y \subseteq \mathbb{N}^{\mathbb{N}}$ is dominating. Is there a Borel set $H \subseteq \mathbb{N}^{\mathbb{N}} \times \mathbb{N}^{\mathbb{N}}$ such that every meager set is contained in $H_{x}$ for some $x \in X \cup Y$ ?

Acknowledgments. We thank Ljubisa Kočinac, Nadav Samet, Marion Scheepers, and Lyubomyr Zdomskyy for their useful comments on this chapter.

\section{REFERENCES}

[1] L. Babinkostova, Metrizable groups and strict o-boundedness, Matematicki Vesnik 58 (2006), 131-138.

[2] L. Babinkostova, Lj. D. R. Kocinac, and M. Scheepers, Combinatorics of open covers (VIII), Topology and its Applications 140 (2004), 15-32.

[3] L. Babinkostova, Lj. D.R. Kočinac, and M. Scheepers, Combinatorics of open covers (XI): Menger- and Rothberger-bounded groups, Topology and its Applications 154 (2007), 1269-1280.

[4] L. Babinkostova, M. Scheepers, and B. Tsaban, Local-global principles in topology (I), unpublished notes.

[5] T. Banakh, P. Nickolas, and M. Sanchis, Filter games and pathological subgroups of a countable product of lines, Journal of the Australian Mathematical Society 81 (2006), 321-350.

[6] T. Banakh and L. Zdomskyy, Separation properties between the $\sigma$-compactness and Hurewicz property, Topology and its Applications 156 (2008), 10-15.

[7] T. Banakh and L. Zdomskyy, Selection principles and infinite games on multicovered spaces and their applications, book in progress.

[8] T. Bartoszyński and H. Judah, Strong measure zero sets, Israel Mathematical Conference Proceedings 6 (1993), 13-62.

[9] T. Bartoszyński and H. Judah, On cofinality of the smallest covering of the real line by meager sets II, Proceedings of the American Mathematical Society 123 (1995), 1879-1885.

[10] T. Bartoszyński and S. Shelah, Continuous images of sets of reals, Topology and its Applications 116 (2001), 243-253.

[11] T. Bartoszyński, S. Shelah, and B. Tsaban, Additivity properties of topological diagonalizations, The Journal of Symbolic Logic 68 (2003), 1254-1260.

[12] T. Bartoszyński and B. Tsaban, Hereditary topological diagonalizations and the Menger-Hurewicz Conjectures, Proceedings of the American Mathematical Society 134 (2006), 605-615.

[13] A. R. Blass, Combinatorial cardinal characteristics of the continuum, in: Handbook of Set Theory (M. Foreman, A. Kanamori, and M. Magidor, eds.), Kluwer Academic Publishers, Dordrecht, to appear.

[14] E. Borel, Sur la classification des ensembles de mesure nulle, Bulletin de la Societe Mathematique de France 47 (1919), 97-125.

[15] J. B. Brown and G. V. Cox, Continuous images of Lusin sets, Proceedings of the American Mathematical Society 89 (1983), 52-54. 
[16] J. Brendle, Generic constructions of small sets of reals, Topology and it Applications 71 (1996), 125-147.

[17] L. Bukovský and J. Haleš, QN-space, wQN-space, and coverings properties, Topology and its Applications 154 (2007), 848-858.

[18] E. K. van Douwen, The integers and topology, in: Handbook of Set Theoretic Topology (eds. K. Kunen and J. Vaughan), North-Holland, Amsterdam: 1984, 111-167.

[19] D. H. Fremlin, Sequential convergence in $C_{p}(X)$, Commentationes Mathematicae Universitatis Carolinae 35 (1994), 371-382.

[20] D. H. Fremlin, SSP and WQN, preprint.

[21] F. Galvin and A. W. Miller, $\gamma$-sets and other singular sets of real numbers, Topology and it Applications 17 (1984), 145-155.

[22] J. Gerlits and Zs. Nagy, Some properties of $C(X)$, I, Topology and its Applications 14 (1982), 151-161.

[23] G. Gruenhage, Products of Fréchet spaces, Topology Proceedings 30 (2006), 475-499.

[24] J. Haleš, On Scheepers' conjecture, Acta Universitatis Carolinae Mathematica et Physica 46 (2005), 27-31.

[25] C. Hernandez, Topological groups close to being $\sigma$-compact, Topology and its Applications 102 (2000), 101-111.

[26] C. Hernandez, D. Robbie and M. Tkačenko, Some properties of $o$-bounded and strictly o-bounded groups, Applied General Topology 1 (2000), 29-43.

[27] W. Hurewicz, Über eine Verallgemeinerung des Borelschen Theorems, Mathematische Zeitschrift 24 (1925), 401-421.

[28] W. Hurewicz, Über Folgen stetiger Funktionen, Fundamenta Mathematicae 9 (1927), 193-204.

[29] F. Jordan, There are no hereditary productive $\gamma$-spaces, Topology and its Applications 155 (2008), 1786-1791.

[30] W. Just, A. W. Miller, M. Scheepers, and P. J. Szeptycki, The combinatorics of open covers II, Topology and its Applications 73 (1996), 241-266.

[31] Lj. D. R. Kocinac, Selected results on selection principles, in: Proceedings of the 3rd Seminar on Geometry and Topology (Sh. Rezapour, ed.), July 15-17, Tabriz, Iran, 2004, 71-104.

[32] Lj. D. R. Kočinac and M. Scheepers, Function spaces and a property of Reznichenko, Topology and it Applications 123 (2002), 135-143.

[33] Lj. D. R. Kočinac and M. Scheepers, Combinatorics of open covers (VII): Groupability, Fundamenta Mathematicae 179 (2003), 131-155.

[34] A. Krawczyk and H. Michalewski, Linear metric spaces close to being $\sigma$-compact, Technical Report $46 \quad$ (2001) of the Institute of Mathematics, Warsaw University. http://www. minuw. edu.pl/english/research/reports/tr-imat/46/products.ps

[35] A. Krawczyk and H. Michalewski, An example of a topological group, Topology and its Applications 127 (2003), 325-330.

[36] M. Machura and B. Tsaban, The combinatorics of the Baer-Specker group, Israel Journal of Mathematics 168 (2008), 125-151.

[37] H. Mildenberger, S. Shelah, and B. Tsaban, The combinatorics of $\tau$-covers, Topology and its Applications 154 (2007), 263-276. 
[38] A. Miller and B. Tsaban, Point-cofinite covers in Laver's model, Proceedings of the American Mathematical Society 138 (2010), 3313-3321.

[39] A. Miller, B. Tsaban, and L. Zdomskyy, Selective properties and products, in preparation.

[40] K. Menger, Einige Überdeckungssätze der Punktmengenlehre, Sitzungsberichte der Wiener Akademie 133 (1924), 421-444.

[41] A. W. Miller, Special subsets of the real line, in: Handbook of Set-Theoretic Topology (K. Kunen and J. E. Vaughan, eds.), North Holland, Amsterdam: 1984, 201-233.

[42] A. W. Miller, A Nonhereditary Borel-cover $\gamma$-set, Real Analysis Exchange 29 (2003/4), 601-606.

[43] T. Orenshtein and B. Tsaban, Linear $\sigma$-additivity and some applications, Transactions of the American Mathematical Society 363 (2011), 3621-3637.

[44] T. Orenshtein and B. Tsaban, Pointwise convergence of partial functions: The Gerlits-Nagy Problem, preprint.

[45] J. Pawlikowski and I. Recław, Parametrized Cichon's diagram and small sets, Fundamenta Mathematicae 147 (1995), 135-155.

[46] R. Pol, A homeomorphism between strong measure zero sets whose graph is not of strong measure zero, Topology and its Applications 157 (2010), 1506-1509.

[47] I. Recław, Every Luzin set is undetermined in the point-open game, Fundamenta Mathematicae 144 (1994), 43-54.

[48] F. Rothberger, Sur des families indenombrables de suites de nombres naturels, et les problémes concernant la proprieté $C$, Proceedings of the Cambridge Philosophical Society 37 (1941), 109-126.

[49] M. Sakai, Special subsets of reals characterizing local properties of function spaces, in: Selection Principles and Covering Properties in Topology (L. Kočinac, ed.), Quaderni di Matematica 18, Seconda Universita di Napoli, Caserta, 2006, 195-225.

[50] M. Sakai, The sequence selection properties of $C_{p}(X)$, Topology and its Applications 154 (2007), 552-560.

[51] M. Scheepers, Combinatorics of open covers I: Ramsey theory, Topology and its Applications 69 (1996), 31-62.

[52] M. Scheepers, Finite powers of strong measure zero sets, Journal of Symbolic Logic 64 (1999), 1295-1306.

[53] M. Scheepers, Sequential convergence in $\mathrm{C}_{p}(X)$ and a covering property, EastWest Journal of Mathematics 1 (1999), 207-214.

[54] M. Scheepers, The length of some diagonalization games, Arch. Math. Logic 38 (1999), 103-122.

[55] M. Scheepers, $C_{p}(X)$ and Arkhangel'skii's $\alpha_{i}$ spaces, Topology and its Applications 89 (1998), 265-275.

[56] M. Scheepers and F. Tall, Lindelöf indestructibility, topological games and selection principles, Fundamenta Mathematicae 210 (2010), 1-46.

[57] M. Scheepers and B. Tsaban, The combinatorics of Borel covers, Topology and its Applications 121 (2002), 357-382.

[58] M. Scheepers, Selection principles and covering properties in topology, Note di Matematica 22 (2003), 3-41. 
[59] S. Shelah and B. Tsaban, Critical cardinalities and additivity properties of combinatorial notions of smallness, Journal of Applied Analysis 9 (2003), 149 162.

[60] P. Simon and B. Tsaban, On the Pytkeev property in spaces of continuous functions, Proceedings of the American Mathematical Society 136 (2008), 11251135 .

[61] B. Tsaban, A topological interpretation of $\mathfrak{t}$, Real Analysis Exchange 25 (1999/2000), 391-404.

[62] B. Tsaban (ed.), SPM Bulletin 1 (2003), http://arxiv.org/math/0301011

[63] B. Tsaban, Selection principles and the minimal tower problem, Note di Matematica 22 (2003), 53-81.

[64] B. Tsaban, The combinatorics of splittability, Annals of Pure and Applied Logic 129 (2004), 107-130.

[65] B. Tsaban, o-bounded groups and other topological groups with strong combinatorial properties, Proceedings of the American Mathematical Society 134 (2006), 881-891.

[66] B. Tsaban, Strong $\gamma$-sets and other singular spaces, Topology and its Applications 153 (2005), 620-639.

[67] B. Tsaban, The Hurewicz covering property and slaloms in the Baire space, Fundamenta Mathematicae 181 (2004), 273-280.

[68] B. Tsaban, Selection principles in Mathematics: A milestone of open problems, Note di Matematica 22 (2003), 179-208.

[69] B. Tsaban, Some new directions in infinite-combinatorial topology, in: Set Theory (J. Bagaria and S. Todorčevic, eds.), Trends in Mathematics, Birkhauser, 2006, 225-255.

[70] B. Tsaban, Additivity numbers of covering properties, in: Selection Principles and Covering Properties in Topology (L. Kočinac, ed.), Quaderni di Matematica 18, Seconda Universita di Napoli, Caserta 2006, 245-282.

[71] B. Tsaban, Menger's and Hurewicz's Problems: Solutions from "The Book" and refinements, Contemporary Mathematics 533 (2011), 211-226.

[72] B. Tsaban and T. Weiss, Products of special sets of real numbers, Real Analysis Exchange 30 (2004/5), 819-836.

[73] B. Tsaban and L. Zdomskyy, Menger's covering property and groupwise density, Journal of Symbolic Logic 71 (2006), 1053-1056.

[74] B. Tsaban and L. Zdomskyy, Scales, fields, and a problem of Hurewicz, Journal of the European Mathematical Society 10 (2008), 837-866.

[75] B. Tsaban and L. Zdomskyy, Combinatorial images of sets of reals and semifilter trichotomy, Journal of Symbolic Logic 73 (2008), 1278-1288.

[76] T. Weiss, A note on unbounded strongly measure zero subgroups of the BaerSpecker group, Topology and its Applications 156 (2008), 138-141.

[77] T. Weiss, On Meager Additive and Null Additive Sets in the Cantor Space $2^{\omega}$ and in $\mathbb{R}$, Bulletin of the Polish Academy of Sciences - Mathematics Series 57 (2009), 91-99.

[78] T. Weiss and B. Tsaban, Topological diagonalizations and Hausdorff dimension, Note di Matematica 22 (2003), 83-92.

[79] L. Zdomskyy, A semifilter approach to selection principles, Commentationes Mathematicae Universitatis Carolinae 46 (2005), 525-540. 
[80] L. Zdomskyy, A semifilter approach to selection principles II: $\tau^{*}$-covers, Commentationes Mathematicae Universitatis Carolinae 47 (2006), 539-547.

[81] L. Zdomskyy, Can a Borel group be generated by a Hurewicz subspace?, Matematychni Studii 25 (2006), 219-224.

Department of Mathematics, Weizmann Institute of Science, ReHOVOT 76100, ISRAEL

Current address: Department of Mathematics, Bar-Ilan University, Ramat-Gan 52900, Israel

E-mail address: tsaban@math.biu.ac.il

$U R L$ : http://www.cs.biu.ac.il/ tsaban 